\title{
Guillain- Barre Syndrome: A Rare Immune Mediated Polyneuropathy
}

\author{
Nama Ravi Sneha Keerthi ${ }^{\star}$, Neelathahalli Kasturirangan Meera \\ Department of Pharmacy Practice, Visveswarapura Institute of Pharmaceutical Sciences, Bengaluru, Karnataka, INDIA.
}

\begin{abstract}
Guillain-Barré Syndrome (GBS) is a rare autoimmune disorder associated with demyelinating polyneuropathy requiring early diagnosis and accurate treatment. From birth to 30 years, the annual incidence is fairly uniform at 1.3 to 1.9 per 100,000 . Clinical hallmarks include symmetrical flaccid muscle paresis and areflexia in the presence of an increased cerebrospinal fluid protein and electrophysiologic studies demonstrating demyelination. A case of 29 year old female presenting to a tertiary care hospital with GB syndrome and hypothyroidism is described. The significance of an appropriate diagnosis by the physician and subsequent management by the clinical staff is reviewed.
\end{abstract}

Key words: Autoimmune, Areflexia, Demyelination, Demyelinating polyneuropathy, Flaccid muscle paresis, Hypothyroidism.

\section{INTRODUCTION}

Guillain-Barré Syndrome (GBS) is an acute inflammatory polyneuropathy with an autoimmune etiology. ${ }^{1}$ It may manifest as limb pain, numbness, weakness, paresthesias, or cranial nerve dysfunction associated with abated or absent deep tendon reflexes. The incidence of GBS is 1.72 patients per 100,000 person-years, with $50 \%$ increase for every 10-year increase in age. ${ }^{2}$ From birth to 30 years, the annual incidence is fairly uniform at 1.3 to 1.9 per $100,000 .^{3}$ Recent studies show that GBS can be classified into 4 main clinical and electrophysiological subtypes such as Acute Inflammatory Demyelinating Polyneuropathy (AIDP), Acute Motor Axonal Neuropathy (AMAN), Acute Sensory Axonal Neuropathy (AMSAN) and Miller Fisher Syndrome (MSF). ${ }^{4}$ GBS can be caused by viral or bacterial infections, including influenza and Campylobacter jejuni. It has also been reported as an adverse event following immunization. The 1976 swine-type influenza-A/ New Jersy vaccine was associated with an excess risk of GBS of 1 per 100,000 vaccines. ${ }^{5}$ When patients present with rapidly progressive paralysis, the diagnosis of Guillain-Barre syndrome needs to be made at the earliest and may require hospital admission for precise monitoring treatment and supportive care. ${ }^{6}$ The diagnosis being challenging, a thorough medical assessment may be required to exclude "mimic disorders" like diphtheria. Nerve conduction studies, CSF analysis and electromyography are significant to establish diagnosis. The goal of treatment is to reduce the severity of the illness and to assist in the patient's recovery. High dose immunoglobulin therapy, physical therapy to increase muscle flexibility and strength and plasmapheresis are notable treatment for GBS. ${ }^{7}$ The case of GB syndrome in a 29 year old female patient is discussed here.

\section{CASE REPORT}

A 29 year old female from South India presented with fever, pain and shaking of lower limbs (calf muscles), pain in upper limbs, difficulty in walking, eating and guiding food to the mouth since 3 days with loss of appetite and she had two episodes of vomiting, non-bile/ blood tinged, containing food particles. Increased pain on walking,
DOI: 10.5530/ijopp.10.3.47

Address for correspondence: Sneha Keerthi, Visveswarapura Institute of Pharmaceutical Sciences, 22nd main, 24th cross, BSK-2nd stage, Bengaluru- 560070, Karnataka, INDIA.

Phone no: +91 9739929736

E-mail: sneha.nr@gmail.com

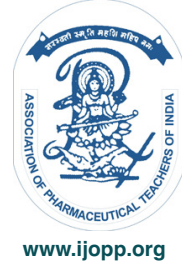


sudden in onset and gradually progressive was reported. Patient noticed shaking of lower limbs when she tried to stand up and walk and was unable to walk without support. No history of similar complaints or trauma was ever reported. She had a past history of hypothyroidism and was treated with $\mathrm{T}$. Thyronorm for 3 months, 4 years ago. Physical examination revealed that she was moderately built, nourished, conscious, co-operative, well oriented to time, place and person. Further examination revealed reduced plantar reflexes, rhomberg's test positive, finger nose test impaired, intention tremors, hypotonia of all 4 limbs and neck rigidity. Deep tendon reflexes and sensory loss was variable in both upper and lower limbs. Abdominal examination, respiratory and cardiac function were found to be normal.

The laboratory findings showed normal blood picture with abnormal $\mathrm{TSH}, \mathrm{T}_{3}$ and $\mathrm{T}_{4}$ values. CSF analysis showed the presence of occasional lymphocytes with good number of RBC's and an elevated protein level of $276 \mathrm{mg} / \mathrm{dL}$ (normal range $<50 \mathrm{mg} / \mathrm{dL}$ ). Electroneuromyography showed axon loss demyelinating neuropathy.

The course of treatment during hospital stay included IV ceftriaxone $2 \mathrm{~g}$ BID $\times 5$ days converted to tab. cefixime + clavulanic acid $200 \mathrm{mg}$ BID $\times 5$ days, IV pantoprazole 40 mg OD, tab paracetamol $650 \mathrm{mg}$ SOS, IV normal saline at $50 \mathrm{ml} / \mathrm{hr}$, IV human normal immunoglobulin (1 vial of $100 \mathrm{ml}=5 \mathrm{~g}$ ), tab. thyronorm $50 \mathrm{mcg}$ OD, injection mecolight 1 amp. In $100 \mathrm{ml} \mathrm{NS}$ OD $\times 8$ days converted to syp. mecolight 4C $10 \mathrm{ml} \mathrm{BID} \times 2$ days. On day $3 \mathrm{CSF}$ aspiration was done for microbiological, cytological and biochemical analysis. This was followed by administration of IV immunoglobulin of 2 vials each on $3^{\text {rd }}$ and $4^{\text {th }}$ day, 3 vials each on $5^{\text {th }}$ and $6^{\text {th }}$ day, 4 vials on $7^{\text {th }}$ day of hospitalization with a test dose given before starting each vial at MICU.

On day 5 and 6, patient showed reaction to human immunoglobulin transfusion and it was de-challenged. The patient was administered with inj. Pheneramine maleate $1 \mathrm{amp}$ stat and tab paracetamol $650 \mathrm{mg}$ SOS and subsequently immunoglobulin transfusion was continued. Except for hypotonia of all left lower limbs, all other parameters showed clinical progress on treatment. Patient was discharged with advice to continue syp. mecolight $10 \mathrm{ml} \mathrm{BID}$ for a month and tab thyronorm $50 \mathrm{mcg}$ OD.

\section{DISCUSSION}

GBS is an acute inflammatory demyelinating polyradiculoneuropathy affecting the peripheral nervous system and a trigger for the same is an acute infection. It can affect all age groups; incidence increases with age, and there is a minor peak among young adults. ${ }^{8}$ Complications like ventilator failure and cardiovascular instability are often observed. This disorder has no cure, but several treatments can ease symptoms and abate the duration of the illness. ${ }^{8}$ Use of steroid hormones have not shown to be beneficial in clinical trials. In the present case, a young adult female patient had history fever and two episodes of vomiting and her total count, differential count and ESR were found to be normal. However, electroneuromyography showed axon loss demyelinating neuropathy. The CSF analysis indicated the presence of lymphage with RBC's and an elevated protein value. These findings meet with the required diagnostic criteria to confirm GBS. Thyroid function tests revealed abnormal levels of TSH. However, no literature states co-relation between thyroid function and GBS. The patient was treated with IV immunoglobulins with a test dose prior to every dose of administration. However, the patient developed fever and rashes which were treated after temporary de-challenge of immunoglobulin. By the end of hospital stay, the patient showed an overall improvement in the movement of all 4 limbs, finger nose test, eating and guiding food to the mouth and walking. Areflexia, a major complaint observed on the day of admission was also resolved. At discharge she was prescribed with a vitamin syrup and thyroid supplementation. The recovery period in general may take few weeks or as long as few years. After few years of the initial attack, about 3\% may suffer a relapse of muscle weakness and tingling sensations. Individuals with GBS also face emotionally painful periods along with physical difficulties. Sudden paralysis and dependence on others for daily activities makes it difficult for them to accept, hence may need psychological counseling to help them adapt. Scientists are working on finding new treatments. GBS after a viral or bacterial infection suggests that certain characteristics of some viruses and bacteria may activate the immune system inappropriately. ${ }^{7}$

\section{CONCLUSION}

GBS is a rare and serious disorder hence it is very important to identify and refer the affected patients to undergo necessary investigation and receive appropriate therapy at the earliest.

\section{ACKNOWLEDGEMENT}

The authors would like to thank the physicians, PG's and 
the working staff of Department of Medicine, KIMS

Bengaluru for imparting required knowledge about the syndrome.

\section{CONFLICT OF INTEREST}

The authors declare no conflict of interest.

\section{ABBREVIATIONS USED}

GBS: Gullain- Barre Syndrome, AIDP: Acute inflammatory demyelinating polyneuropathy, AMAN: acute motor axonal neuropathy, AMSAN: Acute sensory axonal neuropathy, MSF: Miller Fischer syndrome, CSF: Cerebrospinal fluid, TSH: Thyroid stimulating hormone, ESR: Erythrocyte sedimentation rate.

\section{REFERENCES}

1. Suarez IG, Gallego IS, Rivera JR, Arpa J. Guillian-Barre syndrome: natural history and prognostic factors: a retrospective review of 106 cases. BMC Neurology. 2013;13(95):1-6

2. Dubey D, Kapotic M, Freeman M, Sawhney A, Rojas JC, Warnack W, et al. Factors contributing to delay in diagnosis of Guillain-Barre syndrome and impact on clinical outcome. Muscle and Nerve. 2015;1-5.

3. Newswanger DL, Warren CR. Guillian- Barre syndrome. American Family Physician. 2004;69(10):2405-10.

4. Tandel H, Vanza J, Pandya N, Jani P. Guillain- Barre syndrome (GBS): A review. EJPMR. 2016;3(2):366-71.

5. Top KA, Desai S, Moore D, Law BJ, Vaudry W, et al. Guillian- Barre syndrome after immunization in Canadian children. PIDJ. 2015;34(12):1411-3.

6. Leeuwen NV, Lingsma HF, Vanrolleghem AM, Miriam CJ, Sturkenboom M, et al. Hospital admissions, transfers and costs of Guillian- Barre syndrome. Plos One. 2016;11(2):1-12.

7. National Institutes of Health (US). Guillain-Barre syndrome. U.S. department of health and human services public health service; 2011.

8. Pithadia AB, Kakadia N. Guillain-Barre syndrome (GBS). Pharmacological Reports. 2010;62:220-32. 